Relations industrielles

Industrial Relations

\title{
Évolution jurisprudentielle relative aux règles gouvernant la cessation du contrat individuel de travail
}

\section{Alain Turcotte}

Volume 33, numéro 3, 1978

URI : https://id.erudit.org/iderudit/028896ar

DOI : https://doi.org/10.7202/028896ar

Aller au sommaire du numéro

Éditeur(s)

Département des relations industrielles de l'Université Laval

ISSN

0034-379X (imprimé)

1703-8138 (numérique)

Découvrir la revue

Citer cet article

Turcotte, A. (1978). Évolution jurisprudentielle relative aux règles gouvernant la cessation du contrat individuel de travail. Relations industrielles / Industrial Relations, 33(3), 544-563. https://doi.org/10.7202/028896ar

Tous droits réservés @ C Département des relations industrielles de l'Universite Laval, 1978
Ce document est protégé par la loi sur le droit d'auteur. L'utilisation des services d’Érudit (y compris la reproduction) est assujettie à sa politique d'utilisation que vous pouvez consulter en ligne.

https://apropos.erudit.org/fr/usagers/politique-dutilisation/ 


\title{
DROIT DU TRAVAIL
}

\section{ÉVOLUTION JURISPRUDENTIELLE RELATIVE AUX RËGLES GOUVERNANT LA CESSATION DU CONTRAT INDIVIDUEL DE TRAVAIL}

\author{
Alain TuRCoTte
}

L'importance du régime de droit commun en matière de relations de travail est souvent méconnue. Comme le souligne un auteur, en plus des raisons historiques, juridiques (le contrat de travail est le mode normal d'acquisition de la qualité de salarié) et même philosophiques (principe du libéralisme), l'importance de ce régime vient des besoins pratiques:

«Pour les salariés non syndiqués, soit les deux-tiers de la maind'œuvre totale environ, une partie du droit du travail statutaire est sans effet pratique. Pour eux, l'importance réelle du droit commun pour le régime juridique de leur vie de travail dépasse son importance apparente ${ }^{1}$.

Or, il n'y a qu'un petit nombre d'articles dans notre Code civil concernant l'ensemble des problèmes soulevés par la relation entre l'employeur et son employé. Aussi, la jurisprudence sur les articles 1665 a à 1671 C.C. sur le contrat de travail trace-t-elle les repères qui guideront les juristes sur l'interprétation de ces dispositions.

Pour notre part, nous étudierons le phénomène plus particulier de la cessation du contrat de travail. La jurisprudence déterminante sur le sujet a été rendue dans les années trente ${ }^{2}$ où l'on a décidé de s'inspirer des règles du louage de choses pour calculer la longueur du délai-

* TURCOTTE, Alain, avocat à SOQUIP.

1 Claude D'AOUST, Le contrat individuel de travail en droit québécois, Montréal, P.U.M., 1971, p. 1. Il faut noter, par ailleurs, que la majorité de la main-d'œuvre québécoise est non syndiquée malgré les progrès marqués dans le domaine. De $21,6 \%$ en 1960, les effectifs syndiquaux représentaient en 1972, 30,4\% de l'emploi total, pourcentage qui serait d'environ $31 \%$ en 1974. (Source: Annuaire du Québec 1975-75, Québec, Ministère de l'Industrie et du Commerce, 55e édition, 1976, p. 671 et 672). Voir également les remarques faites par C. D'AOUST et A. ROUSSEAU, Mémoire sur le contrat de travail soumis à l'Office de revision du Code civil, Montréal, Département des relations industrielles, 1970, pp. 1-2.

2 À cette époque, la Cour suprême a tranché parmi les diverses solutions antérieures, particulièrement dans: Asbestos Corp. Ltd c. Cook, ....1933] R.C.S. 86; Dupré Quarries Ltd c. Dupré, [1934] R.C.S. 528; Stewart c. Hanover Fire Insurance Company, [1936] R.C.S. 177. 
congé nécessaire. Il sera, par conséquent, intéressant de voir l'évolution de nos tribunaux depuis les récentes années, surtout depuis 1973, date où les dispositions du louage de choses ont été modifiées ${ }^{3}$.

Au surplus, l'examen de ces questions pose le problème de la justification d'un régime de droit commun en relations de travail individuelles ou collectives. Au moment où l'Office de revision du Code civil a presque terminé sa tâche, la question n'est pas sans intérêt.

\section{LE CONTRAT DE DURÉE INDÉTERMINÉE}

La division classique dans l'étude du louage d'ouvrage est celle portant sur la durée de l'engagement puisque les conséquences sur la cessation seront différentes selon que le contrat est d'une durée déterminée ou pour une entreprise bien précise, ou plutôt sans durée définie. Par ailleurs, on retrouvera plus souvent dans l'industrie des engagements de durée indéterminée et ce, à toutes les échelles de la hiérarchie de l'entreprise. Aussi commencerons-nous par ce type de contrat.

\section{Possibilité de mettre fin au contrat en tout temps}

1.1 Principe. Toute personne soumise au régime de droit commun ne bénéfice d'aucune sécurité d'emploi. Pareillement, tout employeur peut s'attendre à ce qu'un de ses employés le quitte n'importe quand. Il va de soi que le premier énoncé est celui qui, en pratique, est le plus important pour la majorité des gens. Donc, pouvoir pour l'employeur de résilier, unilatéralement, le contrat avec, comme restriction, la «nécessité» d'avoir des raisons valables. Cependant, on ne pourra forcer la réintégration de la personne ainsi congédiée sans raison. Comme le disait le juge Rinfret: "Si elle (en l'occurence une compagnie) l'a fait sans raison valable, l'unique sanction efficace de sa contravention est la condamnation aux dommages-intérêts ${ }^{4}$.»

1.2 Le délai-congé et son importance. Face à la situation, il fallait donc une sorte de mesure compensatoire que constitue le délaicongé. Une des meilleures définitions de cette institution est celle donnée par Planiol et Ripert: «Le délai-congé est un délai que doit respecter celui qui prend l'initiative de la rupture; il s'écoule entre le moment où il signifie cette rupture à l'autre partie et le moment où il rompt avec elle toutes relations de travail... Cette institution a pour objet d'éviter à l'autre partie le préjudice résultant de la brusque cessation du travail. ${ }^{5}$ »

Dans un contexte de grand chômage, la signification de ce mécanisme devient plus importante encore. Que ce soit le simple ouvrier ou bien le cadre de l'entreprise, il faudra accorder ce délai de grâce

3 Loi concernant le louage de choses, L.Q. 1974, c. 74, loi sanctionnée le 22 décembre 1973, en vigueur le premier janvier 1974.

4 Dupré Quarries Ltd c. Dupré, [1934] R.C.S. 528, p. 531.

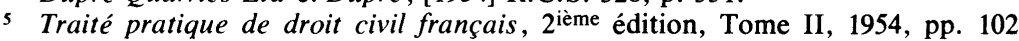
et ss., tel que cité dans Columbia Supplies Co., [1967] B.R. p. 113. 
(très souvent sous forme d'un montant d'argent représentant la période de temps nécessaire). Or, la base de calcul du délai ne fait l'objet d'aucune disposition législative ${ }^{6}$; ce fut la jurisprudence qui se chargea de cette tâche.

1.3 Base de calcul établie par la jurisprudence. C'est en 1933 que la Cour Suprême, faisant le point sur la jurisprudence antérieure, établit quelques règles. En ce qui concerne la manière de calculer le délai-congé, le juge Rinfret s'exprima ainsi «Si l'une des parties trouve le délai insuffisant, il reste au tribunal à apprécier les circonstances et à accorder des dommages-intérêts, s'il en arrive à la conclusion qu'en effet le délai n'a pas été suffisant. Et, sur ce point, l'article $1651 \mathrm{du}$ Code (aujourd'hui 1631 C.C.) pose une règle qui peut servir de guide.$^{7}$ »

L'article 1657 C.C. de l'époque prévoyait un délai de trois mois pour mettre fin à un bail de maison au loyer payable par termes de trois mois ou plus. L'article actuel (art. 1631 C.C.) stipule un avis de un mois ou une semaine, selon que le loyer est payable au mois ou à la semaine. S'il y a un autre terme, l'avis le suivra jusqu'à la limite de trois mois pour les baux excédant un terme de paiement de trois mois. Il s'agit donc, en substance, d'une disposition identique ${ }^{8}$.

A partir de cet arrêt et des précisions apportées dans l'affaire Stewart c. Hanover Fire Insurance Company précitée, une tendance de la jurisprudence, s'inspirant des règles du louage de choses en la matière, se basa sur la période de paiement du salaire pour fixer l'avis nécessaire lors d'un congédiement ${ }^{9}$. Ainsi, une personne payée à chaque semaine, aurait droit à une semaine d'avis, à tous les quinze jours, un avis de deux semaines et ainsi de suite.

En 1949, un amendement au Code civil venait, pour le moins, corriger l'incertitude qui règnait entre l'application intégrale des dispositions du louage de choses et la théorie du délai raisonnable ${ }^{10}$. Le troisième alinéa qu'on ajoutait stipulait: "Dans le cas d'un domestique, serviteur, compagnon ou journalier engagé à la semaine, au mois ou à l'année, mais pour un laps de temps indéfini, il peut être mis fin au contrat par avis, de l'une des parties à l'autre, d'une semaine si l'engagement est à la semaine, de deux semaines si l'engagement est au mois, d'un mois si l'engagement est à l'année.»

6 Sauf le troisième alinéa de l'article $1668 C . C$., ajouté en 1949.

7 Asbestos Corp. Ltd c. Cook, [1933] R.C.S. 86, p. 99.

8 Il n'y aura donc peu de changements si nos tribunaux suivent ce critère. Voir René DOUCET, «La résiliation du contrat de travail en droit québécois», (1974) 9 R.J.T. 249, p. 286.

9 Par exemple: Dorion c. Commissaires du Havre de Québec, (1937) 62 B.R. 92; Concrete Column Clamps c. Pépin, [1949] B.R. 838; Langton c. Dominion Transport Co. Ltd, (1935) 73 C.S. 8; Leclerc c. Cartier Construction Co. Ltd, [1952] C.S. 103: Thibault c. Cie d'autobus de l'Abitibi Ltée, [1952] R.L. 371.

10 (1949) 13 Geo. VI, c. 69, art. 3. Voir les commentaires du juge Rinfret dans Columbia Builders Supplies Co, [1967] B.R. 111, p. 118: 
Cet ajout règlait beaucoup de cas mais avait la faiblesse de ne viser que certaines occupations tout en laissant dans l'ombre les autres professions. De plus, cela pouvait amener des situations absurdes, comme le soulignait le juge Rinfret de la Cour d'Appel: «Je ne puis concevoir qu'un journalier, engagé à l'année mais payé à la semaine, ait droit à un mois d'avis de congé et que le gérant général de l'établissement, également engagé à l'année et payé à la semaine, n'ait droit qu'à une semaine d'avis. » 11

De cette lacune du législateur devaient naître deux théories très voisines: d'une part, l'arrêt Columbia Builders Supplies c. Bartlett posait comme principe la péremption de la théorie de la période de paiement de salaire. À la suite de l'amendement législatif, la Cour d'Appel accorda la primauté à la durée de l'engagement comme base de calcul, interprétant, en pratique, que le législateur désirait que le changement s'applique à tous. On devait donc examiner si la personne était engagé à l'année, au mois, etc.

Plus encore, dans le même arrêt, on consacrait pour «ceux qui occupent un rang dans la hiérarchie des employés» (expression du juge Rinfret) la théorie de l'avis raisonnable par lequel l'ensemble de la situation de la partie frustrée doit être considérée. Cette approche que l'on retrouve particulièrement dans l'affaire Dubois c. J.-René Ouimet Ltée ${ }^{12}$, examinera notamment les circonstances de l'engagement, la nature et l'importance du travail, la difficulté pour se trouver un autre travail, etc.

Par conséquent, depuis ce temps, on peut avancer que la tendance de nos tribunaux à l'application d'un critère fixe, la période de paiement du salaire, est pratiquement disparue. Le manque de souplesse de cette formule n'avait pu tenir le coup devant la protection plus avantageuse que présentaient les lois ouvrières du temps ${ }^{13}$ et, depuis 1964, plus particulièrement, la présence d'un Code du travail concrétisant les droits des travailleurs. C'est donc les changements imposés par une industrialisation massive qui ont provoqué une modification législative et un virage jurisprudentiel.

1.4 Tendances actuelles. Comme on a pu le constater plus haut ${ }^{14}$ il y a une bonne partie de la main-d'œuvre qui n'est pas syndiquée. Nous pourrons voir maintenant de quelle façon les tribunaux se comportent à la lumière des décisions dont nous avons parlé.

1.4.1 Causes de congédiement. Dans un premier temps, nous examinerons parmi les modes généraux d'extinction des contrats de

11 Id, p. 119.

12 [1959] C.S. 573.

13 Voir par exemple les vives critiques des arrêts Concrete Column Clamps Ltd et Dorion c. Commissaires du Havre de Québec, supra, note 9 dans Walter S. JOHNSON, «Lease or Hire of Personal Services - Notice of Termination to Workman - Required Delay - Building Trades», (1950) 28 R. du B. Can. 465.

14 Voir supra, note 1. 
travail ${ }^{15}$, les causes sur lesquelles les juges devront se pencher avant d'adjuger sur le délai-congé. En effet, «... il est important de savoir que le patron peut renvoyer l'employé sans avis s'il a un motif raisonnable et suffisant... (...) Peu importe alors que le contrat soit à durée déterminée ou indéterminée.» 16

Commençons d'abord par les obligations de l'employé en vertu d' contrat de travail. Claude D'Aoust, dans son étude, en relève trois: l'obligation d'exécution, de diligence et de loyauté ${ }^{17}$, René Doucet a adopté les mêmes divisions en les qualifiant de devoir d'obéissance, de respect et de loyauté. Ce sera le plan que nous adopterons.

1.4.1.1 Devoir d'obéissance. Pour donner un exemple de quelques causes récentes traitant de ce devoir, nous prenons le cas de deux employés situés assez bas dans la hiérarchie et d'un autre occupant un poste de cadre. Ainsi dans le cas de Lévesque c. Labrador Welding Construction Ltd $^{18}$, un camionneur travaillant depuis cinq ans dans l'entreprise refusa d'aller chercher les ordures aux domiciles de certains actionnaires de la compagnie lors d'une grève des boueurs. Le contremaître le congédia alors sur le champ. Le juge décida que ce n'était pas une raison valable: «Considérant qu'il était en droit de refuser tout travail qui ne se trouvait pas dans le cadre normal de son travail ${ }^{19}$.

Pareillement, un chauffeur d'autobus scolaire trouva grâce en Cour provinciale de la raison de son congédiement. La commission scolaire invoquait la désobéissance au «Bus Drivers Manual» parce que le chauffeur en question, à la suite d'un accident mineur dont il n'était pas responsable, avait acquiescé à la demande de l'autre automobiliste de ne pas prévenir la police. Le juge décida qu'il ne s'agissait pas d'une faute grave. Dans le dernier cas, la rédactrice en chef d'un prestigieux magazine se vit reprocher d'avoir prolongé indûment ses vacances malgré les directives reçues. Le tribunal, à la lumière de la preuve qui démontrait certains conflits de personnalité, décréta que le tout ne justifiait pas le congédiement ${ }^{20}$.

1.4.1.2. Devoirs concernant l'exécution du travail. Souvent l'évaluation des devoirs de l'employé concernant son emploi sert de prétextes à l'employeur pour congédier facilement. Par exemple, on

15 C. D'AOUST, op. cit., supra note 1, pp. 59 et ss. Ces modes sont: (1) L'accord mutuel des parties, (2) l'inexécution des obligations de la part d'une partie, (3) L'impossibilité d'exécution, (4) La mort d'une partie, (5) La cession de l'entreprise à un tiers, (6) La faillite.

$16 \quad$ Ibid, p. 61.

17 Ibid, supra, note 1, pp. 26 et suivantes. Voir aussi René DOUCET, La résiliation du contrat de travail en droit québécois, Thèse de maîtrise, Université de Montréal, 1974, pp. 65 et suivantes.

18 C.P., Mingan, ${ }^{\circ}$ 650-02-000832-75, le 20 octobre 1976.

19 Id., p. 3.

20 Premier cas: Duranleauc. Eastern Townships Regional School Board, [1977] C.P. 130. Dans le deuxième cas: Plamondon, [1976] C.S. 105 (jugement publié en résumé). 
tirera profit de quelques déficiences dans l'exécution des obligations pour fournir la cause du renvoi. C'est à cette conclusion qu'arriva le juge Gagné dans Chalifour c. Hallmark Automotive Centres Ltd: "Même si le demandeur n'était pas un gérant modèle et, malgré ses quelques défaillances, le tribunal est d'opinion que la défenderesse aurait dû, après l'avoir mis en demeure, d'avoir à changer sa façon d'agir dans un délai imparti...» ${ }^{21}$

Dans une autre affaire ${ }^{22}$, la défenderesse tenta de prouver l'ivresse au travail alors qu'en réalité le véritable motif était une mésentente au sujet du salaire. Par contre, l'employé doit fournir sa prestation. Ainsi, en cas de grève ou de lock-out, on a déjà décidé que l'exceptio non adimpleti contractus pouvait former une opposition efficace à certaines demandes. Dans l'affaire Macdermid et autres c. Atlantic \& Pacific Co. $L t d^{23}$, la Cour supérieure a accepté cette défense puisque les employés qui réclamaient des sommes d'argent à titre de paye de réparation ou "Severance pay» étaient en grève. La compagnie était donc justifiée de fermer ses portes car ses employés n'exécutaient pas leurs obligations.

1.4.1.3. Devoir de loyauté. En dernier lieu cette obligation de fidélité impose à l'employé la tâche de fournir à son employeur le meilleur de lui-même. Il ne pourra utiliser à son profit l'outillage de son patron pour faire des travaux sur son terrain ${ }^{24}$. À l'opposé, l'appréciation de ce devoir confère à quelques employeurs le pouvoir d'inventer un prétexte à congédiement: témoin une affaire où l'on découvrit une «conspiration» contre un gérant général dont aurait été coupable un employé de vingt ans de service ${ }^{25}$. En résumé, les exemples des récentes années ont prouvé rarement une faute caractérisée d'absence de loyauté; en général, ce motif est fréquemment avancé comme raison pour camoufler de mauvaises relations interpersonnelles entre membres de l'entreprise qui se terminent en faveur de ceux qui disposent de l'autorité.

1.4.2. Influences sur le calcul du délai-congé. Il va de soi que les quelques exemples cités plus haut n'ont été mentionné qu'à titre d'illustrations. Néanmoins, ces cas démontrent bien, à défaut d'une tendance certaine, l'absence de sécurité qui menace les non-syndiqués, parfois d'une très longue expérience. Il semble de plus en plus que ce

21 [1976] R.D.T. 586, p. 589.

22 Bouchard c. Anstalt, C.P.M., n ${ }^{\circ}$ 500-02-020010-778, le 21 décembre 1977.

23 [1975] C.S. 54, (jugement publié en résumé). Pour une discussion sur les relations entre le contrat individuel de travail et la convention collective voir aussi Mc Gavin Toastmaster Ltd c. Ainscough, [1976] 1 R.C.S. 718, commentée par Claude D'Aoust dans (1977) 32 R.I. 456-460.

24 Sculnick c. Services Ltd, C.P.M. n 02029774 747, le 17 mai 1976.

25 Louette c. Creditel of Canada Ltd, C.S. $n^{\circ} 500$ 05-002357-745, le 15 décembre 1975: «It is not permissible for an employer to act in the way Desautels did towards Plaintiff, that is, dismissing a long-service employee without compensation».p. 8. Voir aussi Larocque c. Georges Painchaud Inc.., C.P.M. n $\mathrm{n}^{\circ}$ 500-02-006805-761, le 15 février 1978: "Il s'agit en somme, non pas sans doute d'une tempête dans un verre d'eau, mais d'un prétexte futile à un renvoi qui était prémédité...» 
genre d'affaires, à savoir la disproportion entre les actes et la conséquence qu'est le congédiement, qui a amené la disparition du délaicongé basé sur le calcul de la période de paiement du salaire, aura une influence grandissante sur le calcul du délai-congé.

1.4.3. Lutte entre deux tendances. À la lecture des décisions récentes, on constate l'utilisation répétée du terme «délai raisonnable». On ne peut en déduire que la théorie du délai raisonnable est désormais installée dans notre jurisprudence. La plupart du temps, malgré l'emploi de l'expression, on ne prend pas en considération tous les éléments mais on se limite à la durée de l'engagement. Pour aborder ce point, nous utiliserons le critère de la position dans la hiérarchie.

1.4.3.1. Postes élevés. On se souviendra que le juge Rinfret dans l'arrêt Columbia Builders Supplies c. Bartlett ${ }^{26}$ avait accordé presque d'office le bénéfice d'un délai raisonnable aux gens occupant un rang dans la hiérarchie des employés. L'examen des dernières années confirme la validité de cet énoncé. Un des meilleurs exemples de l'application de cette thèse se retrouve dans Plamondon c. Commission Hydro-électrique de Québec ${ }^{27}$; la demanderesse qui était une des artisanes de la première heure de la revue de prestige de la défenderesse, fut congédiée surtout, semble-t-il à cause de conflits de caractère. Par la suite, elle ne put que se trouver des emplois occasionnels de courte durée.

Ceci amena le juge Lawrence Poitras à déclarer:

«Étant donné l'évolution de notre jurisprudence dans ce domaine et en particulier le caractère tout à fait spécial de la profession de la demanderesse, les difficultés encourues par cette dernière malgré ses maintes tentatives à se trouver un emploi régulier et semblable, le tribunal croit que Mlle Plamondon avait droit à un avis de congé de six mois $» 28$.

Il refusa cependant d'accorder le bénéfice du fonds de pension puisqu'il s'agissait en l'occurence non pas d'un droit mais d'une expectative. Un délai de six mois fut également donné dans le cas de Plante c. Ville de Montréal ${ }^{29}$; nous avons là un cas exceptionnel car feu Me Plante obtint par législation le droit de réclamer des indemnités par des congédiements abusifs ${ }^{30}$. Il y avait deux engagements à l'étude; dans le premier qui fut décidé comme contrat de durée indéterminée, la résolution se lisait comme suit: "RESOLU: que monsieur Pacifique Plante soit nommé, à compter du 8 février 1937, secrétaire du leader du Conseil...». ${ }^{31}$

La Cour d'Appel a jugé en l'instance qu'un délai de six mois aurait été requis, délai quant au salaire et aux avantages sociaux. On notera

26 Voir notes 11 et 12.

27 [1976] C.S. 105.

28 Ibid, p. 28 du texte intégral.

29 [1976] C.A. 95 (Jugement publié en résumé.).

30 Loi modifiant la Charte de la Ville de Montréal et la Loi de la Communauté urbaine de Montréal, L.Q. 1973, c. 77, art. 101.2 Loi sanctionnée le 12 avril 1973.

${ }_{31}$ Texte intégral du jugement, p. 3. 
que l'affaire ayant fait l'objet d'enquêtes publiques, la Cour n'élabore pas sur l'ensemble des conditions même s'il est visible qu'on en a tenu compte. Dans la même lignée, monsieur le juge Yvon Jasmin a accordé un délai d'un an à un ingénieur cadre qui avait vu son département fermer et ses chances d'être transféré réduites à néant:

«Tenant compte particulièrement de la nature et de l'importance du travail du demandeur, de la longue durée de son emploi et de la difficulté pour lui de se trouver une position d'égale importance, le tribunal croit que dans ce cas particulier, le délai raisonnable de l'avis de congé devrait être d'un an à compter du 1er décembre $1972 » .{ }^{32}$ [ce

D'autres affaires témoignent de la constance de la jurisprudence à ce sujet: un gérant général de tout un secteur reçoit trois mois d'avis à titre de compensation pour renvoi injustifié $;^{33}$ un ingénieur possédant déjà un emploi stable se vit offrir une somme de $\$ 1500$ par mois pour un projet lui assurant au moins trente mois d'emploi. Dans un premier temps, le juge se référant à l'arrêt Asbestos Corp. Ltd c. Cook (déjà cité) détermina qu'un contrat de "at least 30 months » n'est pas d'une durée déterminée. En second lieu, il repoussa la défense de cas fortuit présentée par la défenderesse en ce qui concernait la fin des travaux car celle-ci devait prévoir le développement de la situation. Un délai de trois mois plus une compensation pour les avantages sociaux fut donc accordé $^{34}$.

1.4.3.2 Postes dits inférieurs. Comme nous l'avons vu, l'énoncé du juge Rinfret semble assez bien suivi dans les cas des personnes possédant un degré dans la hiérarchie ${ }^{35}$. L'étude des jugement concernant les emplois moins élevés expose une légère divergence. En effet, les deux théories modernes se partagent la faveur de nos tribunaux malgré l'emploi généralisé de l'expression «délai raisonnable» qui donnerait à croire que la situation est la même que dans les causes sur les plus hauts salariés.

32 Cohen c. R.C.A. Ltd, [1976] C.S. 1321; dans un cas similaire le juge Pierre Roger a rejeté une demande car le demandeur, surintendant du service de contrôle depuis dix ans, s'était vu offrir un poste similaire par la compagnie qui avait acquis la section de l'entreprise où il travaillait. Il n'avait donc qu'à accepter: Mark c. R.C.A. Ltd, C.P.M. $\mathrm{n}^{\circ}$ 02-022103-779, le 10 février 1978.

${ }^{33}$ Louette c. Creditel of canada Ltd, C.S.M. n ${ }^{\circ} 500$ 05-002357-745, 15 décembre 1975.

34 Bennett c. Fishback \& Moore of Canada Ltd, C.S.M. $\mathrm{n}^{\circ}$ 500-05-006066-748, le 3 juin 1976. Dans une autre affaire Nasr c. Les Industries $H \& H$ Ltée, C.P.M. $\mathrm{n}^{\circ}$ 500-02-042 629-753, le 18 octobre 1977, le juge Hodge disait: "Il reste à déterminer... quel serait le délai juste et raisonnable de l'avis de congé. Il est à noter, d'une part, que le demandeur a été prévenu de son congédiement trois semaines avant son départ et, d'autre part, il y a lieu de considérer que eu égard à l'ampleur de l'entreprise le demandeur occupait un poste relativement important dans cette entreprise...» p. 3 Le juge accorda trois semaines additionnelles.

35 Voir les critiques de R. DOUCET sur cette discrimination, (1974) 9 R.J.T. 286 ss. 
Dans la cause de Périna c. Versailles Ford Sales Ltée ${ }^{36}$, on a donné à un assistant gérant des vente un mois d'avis après avoir évalué la durée de l'engagement. Dans l'affaire, on tint compte du mode de rémunération du demandeur, c'est-à-dire un revenu hebdomadaire et un boni mensuel. Le juge porta également attention aux responsabilités inhérentes au poste. Dans une autre affaire, il s'agissait d'une personne engagée pour une durée indéterminée mais au salaire payé à chaque semaine au prorata du nombre d'heures travaillées. Celle-ci ne bénéficia que d'un avis de quatre heures lors de son congédiement. À cette occasion, M. le juge Guy Tremblay souleva les difficultés du problème: «Le mode de paiement, pas plus que l'incidence du paiement ne saurait être le seul critère devant déterminer la durée de l'engagement. Comment concilier logiquement cette notion de la durée de l'engagement dans un contrat qui a été conclu pour «un laps de temps indéfini $» ?{ }^{37}$

Finalement, après avoir observé que l'article 1668 C.C. ne s'appliquait pas aux employés payés à l'heure, et, d'autre part que la personne en question travaillait depuis 363 jours, le juge décréta qu'une semaine d'avis était requise.

À d'autres occasions, la jurisprudence intègre de plus en plus à la simple considération de la durée de l'engagement les autres éléments tels le genre d'emploi, les responsabilités, la facilité de se trouver un autre emploi, etc. Le prototype par excellence de ces jugements est l'affaire Duranleau c. Eastern Townships Regional School Board ${ }^{\mathbf{3 8}}$ dans laquelle le juge Roberge se penche en premier lieu sur la gravité de l'infraction commise par l'employé, un chauffeur d'autobus. Puis, dans la détermination du délai-congé, le magistrat applique les critères de l'arrêt Dubois, les mêmes critères qui ont été entérinés par le juge Rinfret mais, nous semble-t-il, pour une toute autre catégorie dans la structure de l'entreprise: "C'est en tenant compte de l'âge du demandeur, de la nature du travail qu'il faisait, de son expérience passée et des termes mêmes de l'engagement que j'en arrive à cette conclusion. » ${ }^{39}$

L'expérience se répéta en diverses occasions: un commis voyageur trop âgé au gré de la compagnie reçoit un mois: "Vu le genre d'emploi du demandeur et ses circonstances particulières (...) Il ne s'agit pas en effet d'une position exceptionnelle. Le travail fait par le demandeur n'est pas du genre qui requiert un entraînement et une spécialisation spéciaux $\gg .{ }^{40}$

$36 \quad[1974]$ R.D.T. 590.

37 Tremblay c. L'Aluminium du Canada Ltée, C.P. Roberval, $\mathrm{n}^{\circ}$ 160-32-000215-751, le 3 mai 1976 (Division des petites créances), p. 6 et 7 . Les soulignés sont du juge.

38 [1977] C.P. 130. Voir supra, note 20. «[La Cour] n'apprécie pas que les fautes qu'on lui (au chauffeur) reproche soient suffisamment lourdes pour entraîner un congédiement ». p. 132.

39 Ibid., p. 132.

40 Comeau c. Georges Daigneault Ltée, C.S.M. $\mathrm{n}^{\circ}$ 500-05-009, 727-759, le 12 janvier 1976, M. le juge Ignace-J. Deslauriers à la page 3. 
Un marchand de tabac aura droit à trois mois après examen du contrat, des circonstances de l'engagement, nature du travail, expérience, etc. ${ }^{41}$. Pareillement pour un gérant-adjoint, ancien camionneur, qui perd son poste à la suite de la perte du contrat entre son employeur et la principale requérante des services de l'entreprise, faisant abstraction du paiement du salaire à toutes les semaines, le Tribunal accorda quatre semaines en considération des responsabilités particulières ${ }^{42}$.

\section{Le délai-congé: effets pratiques}

2.1 Point sur la jurisprudence actuelle. Nous donnions précédamment une définition du délai-congé ${ }^{43}$; nous avons également constaté la nécessité de cette institution. Dans l'état actuel des choses, l'incertitude au sujet du moment où une partie mettra fin au contrat impose cette période tampon.

La position de nos tribunaux concernant le calcul de celle-ci semble se préciser si l'on se fie aux récentes années. D'une part, pour le personnel supérieur, la réponse ne fait presqu'aucun doute: le juge, dans l'appréciation du délai nécessaire "doit» tenir compte non seulement de la période totale d'engagement, mais aussi des autres facteurs entourant l'emploi tel que nous l'avons vu plus haut. Ainsi, la nature de la tâche, son degré de difficulté, les démarches qu'elle a occasionnées, la facilité de trouver un emploi ailleurs sont autant d'éléments qui peuvent être pris en considération.

Ce qui est d'avantage surprenant, compte tenu que dans les cas précédents, nos juges s'appuyaient sur les commentaires du juge Rinfret de la Cour d'Appel, c'est le fait de la tendance qui semble se dessiner pour les employés situés plus bas dans la hiérarchie. En effet, on considère de plus en plus l'ensemble des circonstances de la cessation du contrat de même façon que pour les cadres. Il ne s'agirait pas encore d'un mouvement aussi affirmatif mais pourrait indiquer la tendance prochaine de notre jurisprudence. Il y aurait donc uniformité de traitements entre toutes les catégories d'employés, à savoir que la totalité des faits serait examinée dans l'appréciation du délai-congé.

Comme le soulignait un auteur ${ }^{44}$, ne pourrait-on pas avancer qu'il s'agit tout simplement d'un retour aux déclarations du juge Rinfret de la Cour Suprême: «... il reste au tribunal à apprécier les circonstan-

41 Vuhlstock c. Vendel Severs and Norman Severs, C.S.M. $\mathrm{n}^{\circ}$ 500-05-002 740-72, le 21 juillet 1976 .

42 Comtois c. Tétreaultville Transport Inc., C.P.M. $\mathrm{n}^{\circ}$ 02-045775-751, le 3 novembre 1976. Voir également dans le même sens Bouchard c. Anstalt, C.P.M. n ${ }^{\circ}$ 500-02020010-778, le 21 décembre 1977.

43 Définition de Planiol et Ripert, supra, note 5.

44 Donald J. JOHNSTON, «Dismissal Notice in Employment Contracts», (1963) 9 McGill L.J. 138-149. On y retrouvera une analyse pertinente de la jurisprudence d'avant Columbia Builders Supplies Co. "The odds [dans le cas d'une nouvelle décision de la Cour Suprême] are that «reasonable" notice will emerge as the simple answer to an equally simple problem», p. 149. 
ces...» dans l'arrêt Asbestos Corp. Ltd c. Cook de 1933? Il ne faut pas oublier que l'article du louage de choses n'était cité alors qu'à titre de guide. Il semblerait donc que nos cours ont interprété ce renvoi au Code d'une manière un peu trop restrictive en se confinant à la période de paiement du salaire. Peut-être alors que le lent changement jurisprudentiel signifie le retour à la vraie vocation du délai-congé qui est «d'éviter à l'autre partie le préjudice résultant de la brusque cessation du travail» 45

Il est certainement trop tôt pour décréter que ce virage est irréversible ou définitif. Néanmoins, nous nous croyons autorisé par les décisions récentes de dire que le mouvement paraît bien lancé. Il faudrait par conséquent, du côté pratique, que ceux qui désirent cesser le louage d'ouvrage, le plus souvent l'employeur, se basent sur tous les éléments concernant l'emploi. Cette affirmation serait valable, sauf exceptions, à toutes les catégories. De plus, il ne faudrait pas oublier que les délais accordés sont parfois très longs: trois mois, six mois de salaire sont choses du possible. On a même vu l'octroi d'une année complète à titre de dédommagement. Bref, la situation s'est transformée ce qui peut provoquer une incertitude chez certains: il serait peutêtre mieux alors de s'entendre dès la conclusion du contrat sur une période de délai-congé ce qui permettrait d'échapper au droit commun.

2.2 L'avenir du délai-congé. L'Office de révision du Code civil a proposé les mesures suivantes:

«L'avis doit être dans les délais minimums suivants: a) une semaine, deux semaines ou un mois, selon que l'engagement est à tant par semaine, par mois ou par année; b) une semaine, lorsque l'engagement est à la pièce, ou à tant par heure ou par jour, et qu'il a duré six mois de façon continue. Les termes et modalités de paiement du salaire ne modifient pas la nature de l'engagement. Toutefois, le délai peut être majoré lorsque la nature, la durée ou les circonstances particulières de l'emploi le justifient ${ }^{46}$.

Ces propositions rejoignent ce que prédisait Faribault à la suite de l'amendement de 1949 au Code civil ${ }^{47}$. Selon l'Office, il y aurait donc un critère uniforme, un guide objectif applicable à tous avec précision dans le cas d'employés à la pièce, à tant par heure ou par jour. Nous notons

45 Définition de Planiol et Ripert, précité. Nous sommes d'accord avec les remarques de René DOUCET, loc. cit, (1974) 9 R.J.T. 286 et ss, supra, note 35 sur la discrimination dans l'application de la base de calcul, mais, selon les conclusions cluxquelles nous arrivons, il nous semble qu'il y a des correctifs qui sont apportés par l'application plus fréquente de la théorie du délai raisonnable.

46 Texte de l'article 10 du rapport du comité du contrat de travail, Office de révision du Code civil, Montréal, 1969.

47 «... car je crois bien qu'avant longtemps, cette nouvelle règle de l'article 1688 sera appliquée à tous les avis de congé requis pour mettre fin à un louage de services d'une durée indéterminée.» Traité de droit civil du Québec, t. 12 par Léon FARIBAULT, Montréal, Wilson \& Lafleur, 1951, p. 321. Contra: le juge Rinfret dans Columbia Builders Supplies Co., p. 119. 
également que le dernier paragraphe s'inscrit dans la tendance que nous avons cru déceler par l'étude des années récentes: la théorie du délai raisonnable pourra toujours être invoquée devant nos tribunaux pour améliorer le délai selon les circonstances.

Lorsqu'on observe la situation actuelle, il faut reconnaître qu'en général, les parties sont dans le vague en ce qui concerne le délai à accorder. Sauf les cas de l'article 1668 par. 3 C.C., on aura des difficultés à évaluer l'avis nécessaire. D'autre part, il est monnaie courante de ne pas respecter le délai-congé, la période de disponibilité de la part de l'ouvrier et d'obligation de fournir du travail pour l'employeur est toute théorique. En cas d'injustice ou d'insatisfaction, il faudra recourir au processus judiciaire avec tous ses aléas.

Nous sommes donc d'accord avec certaines critiques ${ }^{48}$ émises par des observateurs. On pourrait ainsi prévoir formellement le remplacement de l'avis par une indemnité correspondante, ce qui entérinerait la pratique habituelle. D'autre part, nous hésitons à retenir la suggestion de l'Office dans son approche de la base de calcul du délai (et/ou de l'indemnité) ${ }^{49}$. Les modalités de l'engagement nous semblent inadéquates à traiter des affaires dans ce domaine. Plutôt que de s'inspirer de la période d'engagement (par semaine, par mois, par année), pourquoi ne pas lier l'avis à l'ancienneté de l'employé? On pourrait de cette façon rendre compte avec plus de justice de la part de celui-ci à l'intérieur de l'entreprise. De plus, on pourrait à partir de cette plate-forme «objective» composer avec l'usage des différents milieux, des différents emplois. En dernier ressort, la partie lésée pourrait toujours s'adresser au tribunal qui, par l'emploi de la théorie de l'avis raisonnable, ferait la compensation nécessaire.

En résumé, il faut, dans l'intérêt de tous, trouver un critère assez objectif pour permettre à chacun de déterminer avec passablement de précisions ce à quoi il peut s'attendre en fait d'avis de congé. La solution pour cela, se situe davantage, à notre avis, du côté de l'ancienneté. Il est nécessaire également que nos tribunaux conservent un droit de regard sur le sujet. Si l'on peut y parvenir, on aura facilité de beaucoup la situation de la main-d'œuvre ${ }^{50}$.

48 En particulier avec les opinions de René Doucet dans (1974) 9 R.J.T. 288-289.

49 L'Office se réfère directement à l'article 1668 tel qu'amendé, voir Rapport pp. 18 et 20. Malgré les remarques faites par C. D'AOUST et A. ROUSSEAU dans leur mémoire, supra, note 1, pp. 21 à 23 qui apportent des éléments profitables au débat, par exemple sur la nécessité d'un délai minimum supérieur à une heure, nous soumettons respectueusement une solution différente.

so Sans faire une étude approfondie, il serait bon de noter la situation en France: pour les salariés ayant moins de 6 mois d'ancienneté, il n'y a pas de pré-avis minimum légal; entre 6 mois et 2 ans: un mois minimum; au moins 2 ans: deux mois minimum. Le tout est encadré par les usages, législations et convention particulières. Voir LAMY SOCIAL, sous la direction de F. JULLIEN, Paris, Lamy S.A., 1977, pp. 598 à $606, n^{\circ} 972$ à 989. 


\section{LE CONTRAT DE DURÉE DÉTERMINÉE}

Bien qu'il soit plus courant de retrouver dans l'industrie des contrats de durée indéterminée, on aura à traiter d'ententes conclues pour un terme précis ou une entreprise déterminée. L'article 1667 C.C. est fort laconique: "Le contrat de louage de service personnel ne peut être que pour un temps limité, ou pour une entreprise déterminée. Il peut être continué par tacite reconduction»

C'est à partir de ce texte que les juristes ont dû élaborer l'ensemble du droit applicable. Aussi ne sera-t-on pas surpris de voir dans le domaine une référence particulière aux règles du louage de choses.

\section{Caractéristiques}

Le simple fait de prévoir un cadre à la durée du contrat oblige les parties à le respecter. On ne pourra donc s'en détacher aussi facilement que dans l'autre type de contrat. D'autre part, puisque l'entente devra de toute évidence prendre fin un jour, la question du renouvellement de celle-ci se posera: de quelle façon l'éviter? Dans le cas de reconduction, quelles en seront les modalités?

1.1 Fin du contrat. Nous avons vu précédamment qu'il suffisait d'un avis, d'un délai pour mettre fin au contrat de durée indéterminée, sauf dans les cas où existaient une cause juste et raisonnable qui dispense de l'avis. Nous avons mentionné pareillement les causes générales d'extinction du louage d'ouvrage ${ }^{51}$. Ces grands modes s'appliquent également lorsque la durée est déterminée.

1.1.1 Respect de la durée. Si l'on fait abstraction des causes générales, les parties sont liées par leur accord. La théorie des obligations entre alors en jeu et on ne peut congédier que pour cause:

«Le contrat de louage de service personnel, étant synallagmatique, peut être résilié lorsqu'une des parties ne remplit pas ses obligations, mais les motifs de renvoi par l'employeur avant l'expiration de l'engagement (...) doivent être justifiés, car autrement il devra indemniser l'employé qu'il a congédié.» 52 .

Par exemple, la cause impliquant $\mathrm{Me}_{\mathrm{e}}$ Pacifique Plante déjà citée ${ }^{53}$ illustre ce principe bien établi. Dans un premier temps, la Cour d'Appel étudia la clause d'engagement de celui-ci avec la ville; il s'agit d'une résolution du conseil exécutif du 16 décembre 1954 qui se lit:

«RESOLU: d'approuver la nomination en permanence de $\mathrm{Me}$ Pacifique Plante en qualité d'assistant-directeur du Service de la police et au traitement annuel de $\$ 8500$, y compris le boni de vie chère. ${ }^{54}$

51 Voir supra, note 15

52 M. le juge J-Robert Beaudoin dans Levasseur c. Allard 5-10-15 Ltée, [1972]

C.S. 658, p. 661. Voir aussi Faribault, op. cit., supra, - note 47, p. 314.

53 Supra, note 29.

54 Texte intégral du jugement, p. 8. 
En 1958, l'appelant fut de nouveau congédié (il s'agissait de son deuxième engagement). Face à cette résolution, $m$. le juge Bernier s'appuyant sur la décision Cité de Montréal c. Filion ${ }^{55}$ décida que l'engagement en était un de durée déterminée. La preuve démontra qu'en l'espèce, la ville avait désiré protéger l'appelant des incertitudes d'un emploi d'une durée indéfinie. De plus, en se référant à l'arrêt mentionné, il faut reconnaître qu'un engagement "en permanence» sans signifier un engagement à vie, n'en est pas moins un contrat dont on ne peut se défaire à volonté. On pourrait avancer que le poste devient assuré jusqu'à la retraite sauf raisons valables. En l'occurence le bris de contrat était totalement injustifié, abusif et illégal (nous reprenons les termes du juge).

La situation inverse est certes possible. Un employeur engageant un chauffeur de camion-remorque pour le transport de maisons mobiles a le droit de mettre fin prématurément au contrat d'une durée d'un an lorsque la personne en question subit des accidents démontrant son inaptitude à l'emploi ${ }^{56}$.

En dernier lieu, la Cour d'Appel a mis en relief, malgré un congédiement sans raison valable, que la cessation du contrat doit provoquer des circonstances néfastes pour la personne qui en est victime. S'il n'y a pas de dommages prouvés, on ne pourra rien accorder. Une directrice d'école engagée pour trois ans, dont le poste est aboli quelque temps après, mais qui se voit offrir une charge d'enseignement avec salaire équivalent ne peut se plaindre de son propre refus ${ }^{57}$.

1.1.2 Cessation de plein droit à l'expiration du terme. L'Office de révision propose un article spécifique, se passant de commentaires, établissant légalement cette conséquence «naturelle» du point de vue juridique. Nous tenons quand même à le mentionner à cause de l'impact sur la tacite reconduction.

1.2 Tacite reconduction. Comme dans le bail d'immeubles le contrat, une fois terminé, peut être renouvelé tacitement si les parties conservent le statu quo.

1.2.1 Comment l'éviter. Par le jeu de l'extinction du contrat à l'arrivée du terme, on pourra arrêter la tacite reconduction. Le principe fut exposé en Cour d'Appel, en 1969. Les clauses en litige étaient les suivantes:

«3. The employment of the party of the second part shall commence on September 16, 1963, and shall be for a period of one year;

55 [1969] B.R. 414.

56 Desrochers c. Sirois, C.S.Q. ${ }^{\circ}$ 17-206, le 17 mars 1978, M. le juge Wasson.

57 Commission scolaire du Cap-de-la-Madeleine c. Guillemette, [1976] R.D.T. 25. Cette cause fait le pendant à l'arrêt que nous avons vu en contrat de durée indéterminée Mark c. R.C.A. Ltd, supra, note 32, où l'employé s'était également vu offrir un poste similaire. En temps normal, un bris illégal de louage de services donne lieu à deux sortes de recours: réclamation en salaire échu pourvu que l'employé demeure disponible, ou réclamation en dommages-intérêts. Voir Laferrière c. Larry Faust Realties Co. Ltd, [1971] C.S. 203 et Martel c. Commissaires d'écoles de Wendover, [1961] C.S. 491. 
10. The present agreement shall be renewed automatically from year to year upon its anniversary date, except that, after the elapse of one year, it may be terminated, by either party, upon a prior notice of four months $\gg 58$.

Le 12 août 1964, l'Association qui avait engagé le demandeur selon l'entente ci-haut mentionnée, l'avisa qu'elle ne désirait pas renouveller le contrat qui expirait le 16 septembre 1964. Le demandeur prétendit que la clause numéro 10 obligeait la défenderesse à lui donner quatre mois d'avis. Monsieur le juge Brossard s'exprima en ces termes pour traiter du litige:

«Le mot «renouvellement» appliqué à un contrat de louage de services signifie "la remise en vigueur dans les mêmes conditions» (dictionnaire Robert); par lui-même, ce terme présuppose que le contrat qu'il remet en vigueur a cessé, ne fût-ce que momentanément, d'être en vigueur; il suggère donc une solution de continuité; la remise en vigueur même dite automatique ne peut donc que présupposer que rien n'y fait légalement obstacle. Or, à mon avis, l'avis du 12 août faisait obstacle légal à la remise en vigueur automatique; cet obstacle était l'absence de tout accord, même implicite ou tacite, de la défenderesse à renoncer à l'effet du terme stipulé explicitement. ${ }^{59}$

C'est par ce type de cause, qui établit que le contrat pour être renouvelé ne doit faire l'objet d'aucun avis contraire, que l'importance du principe de fin à l'arrivée du terme revêt toute sa signification. Donc une manifestation évidente de ne pas donner suite au contrat, empêche sa reconduction: l'entente, à son échéance, est terminé.

D'autre part, comme le souligne Faribault ${ }^{60}$ la tacite reconduction repose sur le silence des parties qui fait présumer qu'elles sont d'accord pour continuer. Mais il n'est pas nécessaire d'un avis écrit; on peut tout aussi bien déduire du comportement des parties leur intention de cesser leurs relations. Ainsi, un directeur des programmes, engagé pour un an, avec clause contractuelle d'avis de non-renouvellement de trois mois, cesse son emploi même si l'avis est légèrement tardif. Le fait que son poste eut été confié à un autre, qu'il eut accompli différents travaux en dehors du champ pour lequel il avait été embauché, des absences prolongées, la vente de sa maison, différentes démarches auprès d'autres employeurs, sont autant d'indices que les parties considéraient leur contrat d'ores et déjà terminé. Il n'y avait donc aucune reconduction possible $^{61}$. La réponse sera la même lorsqu'il $\mathrm{y}$ a des discussions qui échouent ${ }^{62}$.

58 Association des architectes de la Province de Québec c. Sarrazin, [1969] B.R. 321, p. 322 .

59 Ibid, p. 324.

60 Op. cit., supra, note 47, p. 68-69.

61 Laberge c. Radio Etchemin Inc., C.S.Q. $n^{\circ}$ 200-05-004517-764, le 2 février 1978, M. le juge Pierre Côté.

62 Roy c. Centre hospitalier Laval et autre, [1976] C.S. 1120, jugement résumé. Il s'agit de l'étude d'un contrat assez complexe entre un médecin et un centre hospitalier universitaire. Néanmoins, on applique la déduction qu'en vertu de discussions qui ont échouées, il ne peut y avoir tacite reconduction sur la "partie remboursable» 
Une dernière illustration de l'empêchement à la poursuite d'une entente est l'impossibilité totale de remplir les obligations. C'est ce qui est arrivé à une institutrice qui travaillait pour une commission scolaire en 1961-62 et 1962-63. Le huit avril 1963, un arrêté-en-conseil provoquait l'annexion de la commission et la séparait entre quatre autres, ce, effectif le 1er juillet 1963. Le 18 mai 1963, la demanderesse recevait une lettre retenant ses services pour l'année scolaire 1963-64. Entretemps, l'école où celle-ci travaillait fut fermée: elle n'enseigna pas de l'année. Devant le tribunal, on tenta d'établir que les quatre commissions ayant failli d'avertir la demanderesse, il y avait eu tacite reconduction et qu'elle avait donc droit au salaire des cinq années réclamées. Monsieur le juge Brossard établit qu'une lettre de la commission exposant les faits et l'arrêté-en-conseil était suffisants comme avertissement ${ }^{63}$.

1.2.2 Modalités. Après l'examen de la fin du contrat comme tel et des moyens pour empêcher la poursuite du bail, il faut aborder l'hypothèse où la reconduction se produit. Il y a longtemps que la Cour Suprême s'est référé aux dispositions du louage de choses par analogie. Ce fut la décision Stewart c. Hanover Fire Insurance Co ${ }^{64}$ qui a établi les critères qui sont toujours suivis de nos jours. Le seul changement d'importance est le changement législatif de $1973^{65}$ qui a modifié le code au sujet du louage de choses. À défaut de jurisprudence récente transformant le courant établi par la Cour Suprême en 1936, il faut donc avancer que la référence au louage de choses est valable et, par ricochet, que les amendements font état de la loi.

C'est l'article 1641 C.C. qui remplace maintenant l'article 1609 c.C. sur lequel se basait la Cour Suprême:

«Le bail est reconduit tacitement pour un an ou pour la même période si celle-ci était originellement inférieure à un an, lorsqu'après l'expiration d'un bail à durée fixe, le locataire continue d'occuper les lieux plus de huit jours sans opposition de la part du locateur. Le bail reconduit est un bail à durée fixe et obéit aux mêmes règles que ce dernier. Il est lui-même sujet à la reconduction. Les dispositions du présent article ne s'appliquent pas au bail d'un local d'habitation régi par les articles 1659 à 1661.»

Cet article précise plusieurs points: si l'employé demeure à l'emploi huit jours après l'échéance du contrat sans opposition, il y a reconduction. Le contrat reconduit est à durée fixe. Donc, il répond aux mêmes conditions ce qui permet l'application de la jurisprudence pertinente mutatis mutandis. Il est lui-même sujet à reconduction. La durée du contrat reconduit est précisée ${ }^{66}$. Voilà en résumé les guides qui de-

du contrat (p. 31 du texte intégral). Par contre, pour la seconde partie du contrat, la présomption soulevée par le silence des parties joue. (p. 32-33).

${ }^{63}$ Lafrenaye c. Commissaires d'écoles pour la municipalité de Chambly, [1975]

C.A. 155 .

[1936] R.C.S. 177. Pour une excellente analyse des conclusions de ce jugement, voir C. D'AOUST, op. cit., supra, note 1, pp. 73-77.

65 L.Q. 1974, c. 74.

66 Voir les commentaires de R. DOUCET, loc. cit., p. 266-267. 
vraient continuer à faire autorité d'autant plus qu'ils sont très clairs et utiles dans le cadre du louage de services.

\section{Prospectives}

Les changements législatifs bien que portant sur un autre domaine ont clarifié de beaucoup les incertitudes engendrées par une référence à des dispositions disparates. En réunissant en un seul article les critères essentiels à la solution des litiges, le législateur a innové peutêtre sans le vouloir. On pourrait certes chicaner sur l'opportunité de se fier à un corps législatif si étranger aux relations de travail. En pratique, toutefois, la jurisprudence n'aura qu'à suivre l'article 1641 C.C. pour devancer le nouveau Code civil.

En effet, les propositions mises de l'avant par L'Office de révision sont quasiment identiques, justement pour dissiper les ambiguités des anciennes dispositions. La seule critique toutefois, porte sur l'absence d'une source de référence à propos du délai pour s'assurer de la reconduction, c'est-à-dire de la période de huit jours de l'article 1641 C.C. ${ }^{67}$ On peut y voir, avec raisons, semble-t-il, un retour en arrière et un élément d'incertitudes inutiles. Bref, il nous paraît que les tribunaux disposent déjà des éléments nécessaires pour rendre des décisions équitables, décisions que les justiciables auront plus de facilité à appliquer dans leurs contrats.

\section{RUPTURE ABUSIVE DU CONTRAT}

Le contrat de travail peut être assorti d'une foule de conditions ${ }^{68}$. Or, on retrouve souvent certaines clauses selon lesquelles l'employeur peut, à volonté, mettre fin au contrat; parfois, il s'agit des deux parties qui s'octroient ce privilège. Ces clauses font échec aux dispositions courantes. La question se pose cependant: peut-on abuser de ce droit contractuel?

\section{La question en droit civil}

En général, on refuse cette thèse de l'abus d'un droit. Notre droit étant fondé en grande partie sur la liberté des personnes, il paraît aberrant qu'on puisse abuser de son exercice. À plus forte raison en matière contractuelle, où selon la théorie classique, il y a des négociations avant d'arriver à l'entente: donc si une clause y est, c'est que les parties l'on voulue. Cependant, la doctrine de l'abus de droit statue de la façon suivante: "The abuse of rights doctrine states essentially that a person

67 Voir le mémoire de D'AOUST et ROUSSEAU, op. cit., supra, note 1, p. 19. Voir aussi R. DOUCET, loc. cit., pp. 268-269.

68 Condition résolutoire simplement potestative, purement potestative, etc. Claude D'AOUST, op. cit., supra note 1, pp. 70 ss. 
may incur civil liability through a certain act, even though such act is within the bounds of a legal right $» 69$.

\section{Approche de la jurisprudence}

Dans ce qu'on peut considérer comme la cause classique sur le sujet $^{70}$, la Cour du Banc du Roi a fait état de la division des juristes sur la thèse de l'abus de droit en matière contractuelle. La clause suivante était à interpréter: "Either party may at any time give written notice to the other party of his or its election to terminate this agreement on sixty days notice... ${ }^{71}$

Le demandeur, vendeur disposant d'une très large autonomie, fut averti que la compagnie désirait modifier son système de gestion. Ceci l'affectait mais la compagnie lui offrit un emploi. Finalement, il déclina l'offre et démissionna. On jugea la clause parfaitement légale, l'exercice du droit de gérance parfaitement normal, aucune malveillance ou malice ne fut prouvée: la demande fut rejetée en appel. L'impression qui se dégageait, malgré l'absence d'une ratio decidendi ferme, était que la théorie ne s'appliquait pas. "N'y a-t-il pas exagération à remettre au juge un contrôle sur l'exercice des droits du créancier et à lui confier la discrétion de décider s'il était utile ou non pour le créancier de les exercer? $\gg 72$.

Par la suite, la Cour du Banc du Roi réitéra sa décision dans SaintLaurent c. Lapointe et Tremblay ${ }^{73}$. Cette fois-ci, la clause autorisait les deux parties à cesser le contrat sans préavis ni compensation. Malgré l'arbitraire du libellé, on jugea la clause légale, compatible avec les bonnes mœurs et l'ordre public. Les jugements subséquents ne révélèrent aucun changement profond, les faits ne démontrant jamais un exercice malicieux et méchant ${ }^{74}$. La théorie de l'abus de droit semblait bien arrêtée.

Depuis ce temps, le législateur nous a habitué à de nombreuses interventions dans les relations entre individus. L'équité dans les contrats (articles 1040a et suivants C.C.), la protection du consommateur, tous ces changements ont entériné davantage la thèse relativiste. En 1971, le juge Monet dans Fiorito c. The Contingency Insurance Co. Ltd ${ }^{75}$

69 David ANG US, «Abuse of Rights in Contractual Matters in the Province of Quebec", (1961-62) 8 McGille L.J. 151.

70 Quaker Oats Co. of Canada Ltd c. Côté, [1949] B.R. 389.

71 Ibid.

72 Antonio PERRAUT, "La critique des arrêts - La théorie de l'abus des droits", (1949) $9 R . d u B .378$.

73 [1950] B.R. 229.

74 Voir Trottier c. McColl Frontenac Oil Co. Ltd, [1953] B.R. 497; Librairie Concorde Ltée c. Ariss, [1961] B.R. 425, p. 426; Houle c. Diamond Taxicab AssociationLtd, [1957] C.S. 56; Ruel c. Banque Provinciale du Canada, [1971] C.A. 343, p. 345 (caractère rigoureux du contrat), etc.

75 [1971] C.S. 1, p. 7. Décision commentée par I.L. BAUDOUIN, "Contratapplication de la théorie de l'abus de droit en matières contractuelles», (1971) $31 R$. du B. 335 et M. TANCELIN, «Abus de droit - Relativité des droits », (1971) 12 C. de D. 220-221. 
a émis un obiter dictum: "Le principe naguère sacro-saint de la force obligatoire des contrats s'effrite non seulement sous l'influence de la jurisprudence mais aussi à la suite de l'intervention du législateur.»

Même si cette opinion a ouvert une porte, la jurisprudence ne nous semble pas encore établie: dans Novio Automobile Inc. c. Mazda Motors Canada $\operatorname{Ltd}^{76}$, le juge Dugas, s'exprimant dans le cadre d'une requête en irrecevabilité, déclare que les arrêts classiques n'ont pas sonné le glas de la théorie de l'abus de droit en matière contractuelle. Or, dans la même affaire le juge Bergeron, sur requête en injonction interlocutoire, paraît plutôt s'inspirer de ces fameux arrêts, pour rejeter la requête toujours avec les mêmes critères: il faut prouver malice ou méchanceté... Le juge Forest peut dire: «Cette théorie de l'abus de droit en matière contractuelle est loin d'être acceptée sous notre droit » ${ }^{77}$.

Malgré tout, la théorie remporte parfois des succès comme dans le cas d'un contremaître qui se plaint de son congédiement et dont l'incompétence et le caractère difficile ne font aucun doute. On souscrit à l'opinion du juge Monet et l'on estime que le demandeur a violé les obligations implicites de son contrat. Il a donc été congédié à bon droit ${ }^{78}$.

\section{Conclusion}

Après revue des jugements sur la question, on peut s'interroger sur le bien fondé de la réticence de nos juges à admettre la théorie de l'abus de droit en matière contractuelle. Ne pourrait-on pas admettre que l'exercice malicieux et méchant d'un droit, bien que rarement prouvé, constitue bel et bien un abus de droit?

«En effet, à partir du moment où le créancier excède son droit, en dépasse les limites, il n'y a plus de droit. Le créancier comrnet un acte malicieux, imprudent, négligent qui paraît étranger au contrat, et engagera sa responsabilité délictuelle ${ }^{79}$.

Cela permettrait peut-être de réconcilier l'irréconciable, de réunir deux courants qui semblent séparés et finalement, dissiper des malentendus. Par exemple, dans l'affaire Rondeau, le juge cite l'avocat de la défenderesse qui apporte un extrait du juge Brossard à son appui comme preuve de l'entrée dans notre droit de cette théorie. Or, dans Cassanne, ces mêmes notes sont utilisées pour confirmer la tendance inverse...

76 [1974] C.S. 385; injonction C.S.M. $\mathrm{n}^{\circ}$ 05-001-811-74, 20 juin 1974 confirmé sur ce point en appel: C.A.M. ${ }^{\circ}$ 09-000634-74, 10 février 1975.

177 Cassanne c. Grolier Ltée, [1976] C.S. 564, jugement en résumé, p. $10 \mathrm{du}$ texte.

78 Rondeau c. Lamarre Valois International Ltée, [1975] C.S. 805.

79 Pierre AZARD, «L'évolution de la doctrine et de la jurisprudence québécoises en matière d'obligations 1948-1958", (1958) $36 R$. du B. Can. 485. Nous souscrivons à l'opinion de Angers LAROUCHE, "Responsabilité civile: Abus de droit Résiliation de contrat", (1975) 35 R. du B. 698-699; aussi dans (1975) 6 R. G. D. 193-275, p. 219-220. Voir aussi A. MAYRAND, "L'abus desdroits en France et au Québec», (1974) 9 R.J.T. 321-340, pp. 336-337. 
De toute façon, il faut bien admettre que le débat est théorique car en pratique le juge analyse les faits et, en s'assurant qu'il n'y a pas eu exercice malicieux ou méchant dans l'exécution des obligations que se sont données les parties dans cette clause de résiliation, il se trouve par le fait même à vérifier qu'aucun des participants ne va à l'encontre ou au-delà de la volonté première des parties. En ce sens, le peu de réussites dans des poursuites du genre n'infirme pas, à nos yeux, la présence «occulte» de la théorie de l'abus de droit dans les stipulations de résiliation du contrat unilatérale ou bilatérale ${ }^{80}$.

\section{CONCLUSION}

L'ensemble de la jurisprudence passée en revue nous a permis de constater d'une part, la souplesse de notre droit civil, capable de traiter des cas les plus divers avec souvent des résultats heureux. Par contre les déficiences du système civil à traiter les relations individuelles de travail d'une façon moderne sont également visibles.

Il est certes surprenant que les contrats de travail dans notre cadre technologique soient encore régis par des décisions datant des années trente. Que ce soit en matière de délai-congé pour les ententes de durée indéterminée, dans le domaine de la reconduction d'un contrat déterminé ou par l'étude des clauses permettant, de manière contractuelle, la brusque rupture du contrat de louage d'ouvrage, une constante s'impose: la lenteur du droit commun à régler des situations qui nécessitent des décisions rapides.

La question qui reste est celle-ci: faut-il enlever ce champ au droit civil ? ${ }^{81}$ Vaut-il rapatrier tout le secteur dans la législation du travail? Voilà le dilemne que devra trancher le législateur...

80 En effet, il est arrivé très souvent que la partie demanderesse avait une mauvaise position de départ: dans Quaker Oats of Canada Ltd, il était en preuve que la compagnie avait fait le maximum d'efforts pour garder l'employé à son service. Dans l'affaire Saint-Laurent, l'employé s'était querellé avec son supérieur. Le juge Jean-Paul Bergeron émit des commentaires sur la moralité "élastique» d'une requérante (Réal Michaud Automobiles Inc. c. Nissan Automobile Co. (Canada) Ltd, [1974] C.S. 561. À d'autres occasions, la clause était tellement précise qu'elle laissait peu de place à une interprétation en faveur d'un abus de droit: Guérard c. Pinkerton's of Canada Ltd, C.P.M. $\mathrm{n}^{\circ}$ 500 02-011834-764, le 11 novembre 1976. On comprendra alors la difficulté au premier abord de convaincre le juge du bien fondé d'une théorie quand les faits sont peu probants.

On notera que 1 absence de motifs sérieux n'a pas été considérée comme abus de droit lors d'un congédiement: l'entreprise s'était prémunie contre les recours en dommages. Mais dans la même affaire, le juge n'a pas accepté la renonciation au boni d'une des parties en faveur de l'entreprise. C'était là aller à l'encontre de l'article 1081 C.C. Le boni était partie intégrante de la rémunération et non une libéralité, la compagnie ne pouvait se dispenser de cette obligation surtout à l'occasion d'un congédiement sans cause: Fortin c. Firestone Tire \& Rubger Company of Canada Ltd, C.S.M. ${ }^{\circ}$ 05-014172-73, le 3 décembre 1976, M. le juge Marc Beauregard. On fait la distinction avec Godbout c. Transport International Pool (Quebec) Ltd, [1975] C.S. 808.

81 R. GAGNON, L. LE BEL; P. VERGE, «Du soi-disant» contrat de travail», (1970) 11 C. de D. 282-302. 